\title{
Development of PREPARE (PREdicting PAtients' Long-Term Outcome for REcovery): A Practically Usable Prediction Model for Quality of Life of ICU Survivors
}

Nina Wubben ( $\nabla$ nina.wubben@radboudumc.nl )

Radboudumc https://orcid.org/0000-0001-6461-0068

Mark van den Boogaard

Radboudumc

Jordache Ramjith

Radboudumc

Laurens LA Bisschops

Radboudumc

Tim Frenzel

Radboudumc

Johannes $G$ van der Hoeven

Radboudumc

Marieke Zegers

Radboudumc

\section{Research}

Keywords: Quality of life, Critical Care, Prediction modelling, Survivors, Critical Care Outcomes, Prognosis

Posted Date: August 12th, 2020

DOI: https://doi.org/10.21203/rs.3.rs-52234/v2

License: (c) (1) This work is licensed under a Creative Commons Attribution 4.0 International License. Read Full License 
The authors have withdrawn this preprint from Research Square 